\title{
NONABSOLUTELY CONVERGENT INTEGRALS IN THE PROBLEM OF RECOVERING THE COEFFICIENTS OF ORTHOGONAL SERIES
}

\author{
VALENTIN SKVORTSOV \\ Department of Mathematics, Moscow State University, Moscow, 119899, Russia \\ E-mail:vaskvor@mech.math.msu.su \\ and \\ Institute of Mathematics, WSP, Plac Weyssenhoffa 11, 85-072 Bydgoszcz, Poland \\ E-mail: skworcow@wsp.bydgoszcz.pl
}

\begin{abstract}
Nonabsolutely convergent Henstock-type integrals are considered. Their application to the problem of recovering the coefficients of orthogonal series is discussed. It is shown in particular that a multiple Haar or Walsh series which converges rectangularly everywhere to a finite function is the Fourier series of its sum in the sense of Henstock-type integral defined with respect to the dyadic basis. In the case of a multiple trigonometric series the same problem of recovering the coefficients by generalized Fourier formulas can be solved by iterated approximate symmetric Henstock integral.
\end{abstract}

1. Introduction. There are some areas in analysis which require integration processes more powerful than Lebesgue integration. In particular this is related to the problem of recovering the coefficients of orthogonal series from their sums. This problem is a generalization of the uniqueness problem for the coefficients of orthogonal series, and it makes sense to consider this problem of recovering only for those orthogonal systems for which the uniqueness theorem is already established. If the coefficients of an orthogonal series are uniquely determined by its sum, then it is natural to expect that they may be recovered from the sum by Fourier formulas, as in the simplest cases, for example in the case of the uniform convergence. Indeed for many known systems (trigonometric, Haar, Walsh, Vilenkin systems) it is true that every series with respect to those systems convergent everywhere to a summable function is the Fourier series of this function. But

2000 Mathematics Subject Classification: 42A63, 42C10, 26A39, 42B05.

Key words and phrases: Henstock integral, derivate basis, trigonometric series, Walsh series, Haar series, rectangular convergence.

This work was supported by RFFI (02-01-00420, 00-15-96143).

The paper is in final form and no version of it will be published elsewhere. 
the point is that the sum of an everywhere convergent orthogonal series can fail to be Lebesgue integrable. For example, it is known (see [37]) that the series

$$
\sum_{k=2}^{\infty} \frac{\sin k x}{\ln k}
$$

converges everywhere but fails to be the Fourier-Lebesgue series. This kind of example can be given for the other systems mentioned above as well. To integrate such series, one needs nonabsolutely convergent integrals. In the cases where the sum is integrable in one or other known general sense, the question is whether the coefficients can be determined by Fourier formulas in which the integral is understood in the same particular sense. The complete solution of the problem of recovering the coefficients of a convergent series with respect to some system is found if a general process of integration is developed so that any everywhere convergent series with respect to the considered system is the Fourier series of its sum in the sense of the defined integral.

In the trigonometric case the first solution of the problem of defining an integral so that the sum function $f$ of an everywhere convergent series

$$
\frac{a_{0}}{2}+\sum_{k=1}^{\infty} a_{k} \cos k x+b_{k} \sin k x
$$

is integrable and the coefficients $a_{k}$ and $b_{k}$ can be written as Fourier coefficients of $f$ is due to Denjoy. He introduced in [7] a very complicated definition of a second order integral called the totalization $T_{2 s}$, which recaptures a function from its second Riemann symmetric derivative. The difficulty of the $T_{2 s}$-totalization which involves a transfinite sequence of operations led other authors to look for an easier solution of the coefficients problem.

Marcinkiewicz and Zygmund [17] were the first to produce a Perron type $M Z$-integral to solve this same question. They where followed by Burkill with his $S C P$-integral [6] and by James [14] with $P^{2}$-integral. The $M Z$-integral turned out to be more general than $S C P$ - and $P^{2}$-integrals (see [24]). The application of all those integrals to the coefficients problem is based on Riemann theory and on reducing the problem to the one of recovering a function from its second order Riemann symmetric derivative. The latest step in this direction was done by Preiss and Thomson [20] who produced a first order integral that itegrates approximate symmetric derivatives.

The problem of recovering the coefficients was also considered for other classical orthogonal systems (see [23], [25], [29], [16]).

Nowadays the unifying approach to this problem, including the multidimensional case, is the reduction of the problem to the one of recovering the function from its derivative with respect to an appropriate derivate basis. To solve the latter problem a Henstock-type integral associated with respective basis is to be defined and applied.

In the first part of this survey we consider Henstock approach to defining integrals and review some new results related to the Henstock theory of integration. In the second part we examine an application of those generalized integrals of Henstock type to the above mentioned problem of recovering the coefficients of orthogonal series. 
2. Henstock-type integrals with respect to different derivate bases. A generalized Riemann integral which turned out to be equivalent to the Denjoy-Perron integral was introduced independently by J. Kurzweil in [15] and R. Henstock in [11] and [12] to integrate real-valued functions defined on an interval of the real line. The idea of the definition is very simple and is based on replacing in the definition of the Riemann integral a positive constant $\delta$ which regulates the length of the intervals constituting a partition, with a positive function $\delta$ defined on the interval of integration.

These original papers written in the late fifties gave rise to a general theory of nonabsolutely convergent integrals. A unifying notion in this theory is that of derivate basis (or differentiation basis). It is usually defined in the classical abstract derivation theory as a family of sets contracting to a point (see [9]). In the theory we are discussing here, a somewhat more subtle definition is needed (see [33], [18], [13]).

We introduce first some notations. If $E \subset \mathbf{R}^{m}$ then $|E|$ denotes the Lebesgue measure of $E$. The terms "almost everywhere" (br. a.e.) and "measurable" are always used in the sense of the Lebesgue measure. In $\mathbf{R}^{m}$ we shall use the maximum norm $\|\mathbf{x}\|=$ $\max _{1 \leq i \leq n}\left|x_{i}\right|$, so that a $\delta$-neighborhood of $\mathbf{x} \in \mathbf{R}^{m}$ denoted by $U(\mathbf{x}, \delta)$ is an open cube centered at $\mathbf{x}$ with sides equal to $2 \delta$. An interval in $\mathbf{R}^{m}$ is a compact set

$$
\left[a_{1}, b_{1}\right] \times\left[a_{2}, b_{2}\right] \times \ldots \times\left[a_{m}, b_{m}\right] \text { with } a_{i}<b_{i}, i=1,2, \ldots, m .
$$

A collection of intervals is called nonoverlapping whenever their interiors are disjoint.

Let $\mathcal{I}$ be the family of all intervals in $\mathbf{R}^{m}$. A nonempty family $\mathcal{B}$ of subsets of the product $\mathcal{I} \times \mathbf{R}^{m}$ will be termed a derivate basis (or simply a basis) on $\mathbf{R}^{m}$. We assume that all bases $\mathcal{B}$ are filtering down, i.e., for every $\beta_{1}, \beta_{2} \in \mathcal{B}$ there exists a $\beta \in \mathcal{B}$ such that $\beta \subset \beta_{1} \cap \beta_{2}$ (let us agree that $\mathcal{B}$ does not contain the empty set). We shall refer to the elements $\beta$ of $\mathcal{B}$ as basis sets. In this paper we shall always suppose that $(I, \mathbf{x}) \in \beta$ implies $\mathbf{x} \in I$, although it is not the case in the general theory.

Given a basis $\mathcal{B}$, an interval $I$ is called a $\mathcal{B}$-interval if $(I, \mathbf{x}) \in \beta$ for some $\mathbf{x}$ and some $\beta \in \mathcal{B}$.

For a set $E \subset \mathbf{R}^{m}$ and $\beta \in \mathcal{B}$ we write

$$
\beta(E)=\{(I, \mathbf{x}) \in \beta: I \subset E\} \text { and } \mathcal{B}[E]=\{(I, \mathbf{x}) \in \beta: \mathbf{x} \in E\} .
$$

All the bases $\mathcal{B}$ we shall consider in this paper are so-called Vitali bases, i.e., such that for any $\mathbf{x}$ and for any basis set $\beta \in \mathcal{B}$ the set $\beta[\{\mathbf{x}\}]$ contains a pair $(I, \mathbf{x})$ with $I$ of arbitrary small diameter.

The simplest derivate basis on $\mathbf{R}^{m}$ is the full interval basis. In this specific case, each basis set corresponds to a positive function $\delta$ defined on $\mathbf{R}^{m}$ and called a gage. For a given gage $\delta$, we denote

$$
\beta_{\delta}=\{(I, \mathbf{x}): I \in \mathcal{I}, \mathbf{x} \in I \subset U(\mathbf{x}, \delta(\mathbf{x}))\} .
$$

So the full interval basis is the family $\left\{\beta_{\delta}\right\}$ where $\delta$ runs over the set of all possible gages.

Beside full interval basis, we shall consider some other interval bases which can be defined using only intervals satisfying some additional properties. For example, if in the definition of the full interval basis we replace arbitrary intervals with intervals (2) subject 
to the regularity condition

$$
\frac{\min _{i}\left(b_{i}-a_{i}\right)}{\max _{i}\left(b_{i}-a_{i}\right)} \geq r
$$

with some fixed $r, 0<r \leq 1$, then we get the $r$-regular interval basis. If we denote the family of all the $r$-regular intervals by $\mathcal{I}_{r}$ then basis sets of the $r$-regular basis are defined by gages as

$$
\beta_{\delta}^{r}=\left\{(I, \mathbf{x}): I \in \mathcal{I}_{r}, \mathbf{x} \in I \subset U(\mathbf{x}, \delta(\mathbf{x}))\right\} .
$$

Another important example of a basis which we shall use here is the dyadic basis $\mathcal{B}_{\mathrm{d}}$. The basis sets of $\mathcal{B}_{\mathrm{d}}$ consist of pairs $(I, \mathbf{x})$, where intervals $I$ are dyadic, i.e, they are Cartesian products of one-dimensional dyadic intervals $I=\left[j / 2^{k},(j+1) / 2^{k}\right]$, with some integers $j$ and $k$. Denoting the family of all the dyadic intervals by $\mathcal{D}$ and using gages we define basis sets of $\mathcal{B}_{\mathrm{d}}$ as

$$
\beta_{\delta}^{d}=\{(I, \mathbf{x}): I \in \mathcal{D}, \mathbf{x} \in I \subset U(\mathbf{x}, \delta(\mathbf{x}))\} .
$$

We get the dyadic r-regular basis if we define basis sets as

$$
\beta_{\delta}^{d r}=\left\{(I, \mathbf{x}): I \in \mathcal{D} \cap \mathcal{I}_{r}, \mathbf{x} \in I \subset U(\mathbf{x}, \delta(\mathbf{x}))\right\} .
$$

We shall also need the definition of the symmetric approximate basis. We confine ourselves here to the one-dimensional case. To define this basis, we use the following auxiliary notion. By a density gage $\gamma$ on $\mathbf{R}$ we mean a measurable subset of the plane $\mathbf{R}^{2}$ so that for each $x \in \mathbf{R}$ the set $\gamma_{x}=\{h: h>0,(x, h) \in \gamma\}$ has inner density 1 on the right at 0 . When a density gage is fixed, we put $\beta_{\gamma}^{s}=\{([x-h, x+h], x):(x, h) \in \gamma\}$. All such basis sets constitute the symmetric approximate basis $\mathcal{B}_{\text {s.ap }}$.

A finite collection $\pi \subset \beta$ is called a $\beta$-partition if for distinct elements $\left(I^{\prime}, x^{\prime}\right)$ and $\left(I^{\prime \prime}, x^{\prime \prime}\right)$ in $\pi$, the intervals $I^{\prime}$ and $I^{\prime \prime}$ are nonoverlapping. If a partition $\pi=\left\{\left(I_{i}, x_{i}\right)\right\} \subset$ $\beta(I)$ for some $I \in \mathcal{I}$ is such that $\cup_{i} I_{i}=I$, then we say that $\pi$ is a $\beta$-partition of $I$.

We say that a basis $\mathcal{B}$ has the partitioning property if for any $\mathcal{B}$-interval $I$ and for any $\beta \in \mathcal{B}$ there exists a $\beta$-partition of $I$. The partitioning property is not so trivial as it may seem to be at first glance. Whereas in the particular case of the full interval basis on $\mathbf{R}$, this property has long been known as the Cousin lemma, in the multidimensional case for some bases it was proved only recently (see [8]), and for some bases the property is not valid at all or holds true only in some weaker sense as in the case of the symmetric approximate basis (see [20], [35]). The weaker form of the partitioning property for the basis $\mathcal{B}_{\text {s.ap }}$ is the following.

Proposition 1. For every basis set $\beta \in \mathcal{B}_{\text {s.ap }}$ there is a set $N \subset \mathbf{R}$ of measure zero such that for every interval with endpoints in $\mathbf{R} \backslash N$ there exists a $\beta$-partition of this interval.

Given a basis $\mathcal{B}$, an interval function $\tau$ defined at least on all $\mathcal{B}$-intervals, a set $E \subset \mathbf{R}^{m}$ and a basis set $\beta$, we denote

$$
\operatorname{Var}(\beta, \tau, E)=\sup \sum_{(I, x) \in \pi}|\tau(I)|,
$$

where "sup" is taken over all $\pi \subset \beta[E]$, and we call it the $\beta$-variation of $\tau$ on the set $E$. 
Then we define

$$
V_{\tau}(E)=\inf \operatorname{Var}(\beta, \tau, E),
$$

where "inf" is taken over all basis sets $\beta \in \mathcal{B}$.

We call $V_{\tau}$ the variational measure generated by $\tau$ with respect to the basis $\mathcal{B}$. Note that $V_{\tau}$ is a metric outer measure on $[a, b]$ (see [34]) and so its restriction to the Borel sets is a measure.

Definition 1. A variational measure $V_{\tau}$ is called absolutely continuous (with respect to the Lebesgue measure) on a set $E$ if $|N|=0$ implies $V_{\tau}(N)=0$ for a set $N \subset E$.

The upper derivative of a $\mathcal{B}$-interval function $\tau$ at a point $x$ with respect to the basis $\mathcal{B}$ is defined as

$$
\bar{D}_{\mathcal{B}} \tau(x)=\inf _{\beta} \sup \left\{\frac{\tau(I)}{|I|}:(I, x) \in \beta[\{x\}]\right\} .
$$

Similarly, the lower derivative is defined as

$$
\underline{D}_{\mathcal{B}} \tau(x)=\sup _{\beta} \inf \left\{\frac{\tau(I)}{|I|}:(I, x) \in \beta[\{x\}]\right\} .
$$

If $\underline{D}_{\mathcal{B}} \tau(x)=\bar{D}_{\mathcal{B}} \tau(x) \neq \pm \infty$, we say that $\tau$ is $\mathcal{B}$-differentiable at $x$ and the derivative is denoted by $D_{\mathcal{B}} \tau(x)$. We recall the definition of Henstock-type integral with respect to a basis $\mathcal{B}$ (see [18], [13]). In this definition we assume that $\mathcal{B}$ has the the partitioning property.

Definition 2. A function $f$ defined on a $\mathcal{B}$-interval $J$ is said to be $H_{\mathcal{B}}$-integrable on $J$ with integral $A$ if for every $\varepsilon>0$ there exists a basis set $\beta$ such that

$$
\left|\sum_{(I, x) \in \pi} f(x)\right| I|-A|<\varepsilon,
$$

for any $\beta$-partition $\pi$ of $J$. We write $A=\left(H_{\mathcal{B}}\right) \int_{J} f$.

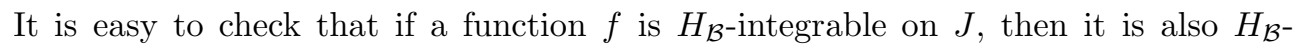
integrable on each $\mathcal{B}$-interval $I \subset J$. Therefore the indefinite $H_{\mathcal{B}}$-integral $F(I)$ is defined as an additive interval function at least on the family of all $\mathcal{B}$-intervals $I \subset J$.

The following useful descriptive characterization of the indefinite $H_{\mathcal{B}}$-integral was established recently (see [3], [4], [2], [28], [36]) for some bases, including the regular basis and regular dyadic basis defined above: $A n$ additive $\mathcal{B}$-interval function $F$ is the indefinite $H_{\mathcal{B}}$-integral of some integrable function if and only if $F$ generates an absolutely continuous variational measure $V_{F}$.

As the symmetric approximate basis has only a weakened form of the partitioning property, a Henstock-type integral with respect to this basis has a slightly different definition. We present here, following [20], a special case of the symmetric approximate integral - the so-called periodic $S A$-integral - which is wide enough to solve the problem of recovering the coefficients of trigonometric series.

We use Proposition 1 . For a basis set $\beta \in \mathcal{B}_{\text {s.ap }}$ we take a set $N \subset \mathbf{R}$ of measure zero such that for every interval with endpoints in $\mathbf{R} \backslash N$ there exists a $\beta$-partition of this interval. Let $N_{1}=N-2 \pi$. The set $M=(\mathbf{R} \backslash N) \cap\left(\mathbf{R} \backslash N_{1}\right)$ is of full measure on $\mathbf{R}$. It is 
clear that for any $x_{0} \in M$ one can find a collection of points $x_{0}<x_{1}<\ldots<x_{n}=x_{0}+2 \pi$ so that

$$
\left(\left[x_{i-1}, x_{i}\right], \frac{x_{i-1}+x_{i}}{2}\right) \in \beta, \quad i=1,2, \ldots, n,
$$

i.e., the collection (3) constitutes a partition of the interval $\left[x_{0}, x_{0}+2 \pi\right]$ by elements of $\beta$. This observation enables the following definition.

Definition 3. Let a function $f: \mathbf{R} \rightarrow \mathbf{R}$ be $2 \pi$-periodic. We say that $f$ has a periodic symmetric approximate integral ( $S A$-integral) if there is a number $A$ such that for every $\varepsilon>0$ there exists $\beta \in \mathcal{B}_{\text {s.ap }}$ such that

$$
\left|\sum_{i=1}^{n} f\left(\frac{x_{i-1}+x_{i}}{2}\right)\left(x_{i}-x_{i-1}\right)-A\right|<\varepsilon,
$$

for any partition by elements (3) of any interval of length $2 \pi$ for which such a partition exists. We write $A=(S A) \int_{0}^{2 \pi} f$.

Note that the limits of integration in this notation are used just to indicate the period but not the fixed interval $[0,2 \pi]$.

Henstock integral with respect to any basis can be given an equivalent Perron-type definition (see [18]), where major and minor functions are defined by lower and upper derivatives with respect to that basis. In this connection we mention some new results related to the known Marcinkiewicz theorem (see [22]) stating that in the case of ordinary Perron integral a measurable function is Perron integrable if it has a pair of continuous major and minor functions. This theorem was extended to some generalized Perron integrals (see [5], [29]). And at the same time it was shown that Perron integral with respect to the symmetric basis and dyadic basis does not have the Marcinkiewicz property (see $[31],[27])$.

3. Solution of the problem of recovering the coefficients. We consider here the problem of recovering the coefficients for multidimensional orthogonal series.

We start with series with respect to Haar and Walsh systems for which the dyadic Henstock integral gives a solution of this problem. These orthogonal systems are of interest from many points of view. In many applications related to signal processing, it has turned out that such systems representing rectangular waves rather than sinosoidal ones, appear to be preferred in a number of cases. Moreover, the Haar system is the simplest example of wavelets and the Walsh system can be interpreted as a group of characters of some totally disconnected Abelian group providing an important model on which one can verify and illustrate many questions from abstract harmonic analysis. We recall the definitions of these systems. For details see [10], [25].

Using the dyadic intervals

$$
\Delta_{j}^{k}=\left(\frac{j}{2^{k}}, \frac{j+1}{2^{k}}\right), \quad 0 \leq j \leq 2^{k}-1, \quad k=0,1,2, \ldots,
$$

we define the Haar system $\left\{h_{n}\right\}$ on the unit interval $[0,1]$ in the following way. Let 
$h_{0}(x)=1$ for all $x \in[0,1]$. If $n=2^{k}+j, j=0, \ldots, 2^{k}-1$ we put

$$
h_{n}(x)=\left\{\begin{aligned}
2^{k / 2} & \text { if } x \in \Delta_{2 j}^{k+1}, \\
-2^{k / 2} & \text { if } x \in \Delta_{2 j+1}^{k+1}, \\
0 & \text { if } x \in[0,1] \backslash \Delta_{j}^{k} .
\end{aligned}\right.
$$

At 0 and 1 the functions $h_{n}$ are defined by continuity from inside of $(0,1)$, and at the points where the Haar functions are left undefined up to now let them be equal to the average of their left and right limits.

To define the Walsh functions we use dyadic expansions of natural numbers as well as those of real numbers in the half-open interval [0,1). Let $n=\sum_{j=0}^{\infty} \varepsilon_{j} 2^{j}$ with $\varepsilon_{j}=0$ or 1 , and $x=\sum_{j=0}^{\infty} x_{j} 2^{-j-1}$ with $x_{j}=0$ or 1 , with a stipulation that for the dyadic rationals we use only finite expansions. With this notation we put

$$
w_{n}(x)=(-1)^{\sum_{j=0}^{\infty} \varepsilon_{j} x_{j}}, \quad n=0,1,2, \ldots
$$

To turn to the multidimensional case let $Z_{0}$ be the non-negative integers and $m$ be the dimension. Then for $\mathbf{k}=\left(k_{1}, \ldots, k_{m}\right) \in Z_{0}^{m}$ we use $\left\||\mathbf{k} \||\right.$ to denote $\min _{i} k_{i}$.

The $m$-dimensional Haar series can be written as

$$
\sum_{\mathbf{n}} a_{\mathbf{n}} \prod_{i=1}^{m} h_{n_{i}}\left(x_{i}\right)=\sum_{n_{1}=0}^{\infty} \ldots \sum_{n_{m}=0}^{\infty} a_{n_{1}, \ldots, n_{m}} \prod_{i=1}^{m} h_{n_{i}}\left(x_{i}\right)
$$

where $\mathbf{n}=\left(n_{1}, \ldots, n_{m}\right) \in Z_{0}^{m}$ and $\mathbf{x}=\left(x_{1}, \ldots, x_{m}\right)$ belongs to the unit interval of $\mathbf{R}^{m}$.

We say that this series is rectangularly convergent to a function $f(\mathbf{x})$ if its rectangular partial sums

$$
S_{\mathbf{k}}(\mathbf{x})=\sum_{n_{1}=0}^{k_{1}} \ldots \sum_{n_{m}=0}^{k_{m}} a_{\mathbf{n}} \prod_{i=1}^{m} h_{n_{i}}\left(x_{i}\right)
$$

are convergent to $f(\mathbf{x})$ when $\||\mathbf{k} \|| \mid \rightarrow \infty$.

We say that the series (4) is r-regular rectangularly convergent, $0<r \leq 1$, to a function $f$ at a point $\mathbf{x}$ if in the previous definition we consider only those rectangular sums (5) for which $\min _{i} k_{i} / \max _{i} k_{i} \geq r$.

In a similar way we define the $m$-dimensional Walsh series and its rectangular and $r$-regular rectangular convegence.

The crucial step to the solution of the coefficients problem for the Haar and Walsh series is to observe that the integral $\int_{\Delta_{\mathbf{j}}^{\mathbf{k}}} S_{2^{\mathbf{k}}}$ where $\mathbf{k}=\left(k_{1}, \ldots, k_{m}\right), \mathbf{j}=\left(j_{1}, \ldots, j_{m}\right)$, $\Delta_{\mathbf{j}}^{\mathbf{k}}=\Delta_{j_{1}}^{k_{1}} \times \ldots \times \Delta_{j_{m}}^{k_{m}}, 2^{\mathbf{k}}=\left(2^{k_{1}}, \ldots, 2^{k_{m}}\right)$, defines an additive interval-function $\psi(\Delta)$ on the family $\mathcal{D}$ of the dyadic intervals and for this function

$$
S_{2^{\mathbf{k}}}(\mathbf{x})=\frac{\psi\left(\Delta_{\mathbf{j}}^{\mathbf{k}}\right)}{\left|\Delta_{\mathbf{j}}^{\mathbf{k}}\right|} \text {, where } \mathbf{x} \in \Delta_{\mathbf{j}}^{\mathbf{k}} .
$$

It follows from this formula that if the Haar or Walsh series is rectangularly convergent (respectively $r$-regular rectangularly convergent) at a point $\mathbf{x}$ with irrational coordinates, then the function $\psi$ is differentiable (respectively $r$-regular differentiable). This implies the following theorem on recovering the coefficients of Haar and Walsh series (see [26]). 
THEOREM 1. If the Haar series (4) is convergent rectangularly everywhere on the unit interval of $\mathbf{R}^{m}$ to a finite function $f$ then it is the Fourier series of $f$ in the sense of $H_{\mathcal{B}}$-integral with respect to the dyadic basis $\mathcal{B}_{\mathrm{d}}$.

A similar theorem holds for multidimensional Walsh series. In the case of $r$-regular rectangular convergence analogous theorems for Haar and Walsh series are also true if we use Henstock integral with the $r$-regular dyadic basis and put some additional conditions on the behaviour of the partial sums at the points with dyadic-rational coordinates.

Now we consider the trigonometric case. As it was already mentioned, symmetric approximate integral ( $S A$-integral) solves the coefficients problem in the one-dimensional case. This solution can be formulated as follows (see [20]).

THEOREM 2. Let the trigonometric series (1) be convergent everywhere to a finite value $f(x)$. Then $f$ is $S A$-integrable on the period, and the series is the SA-Fourier series for $f$, i.e., for each $n$

$$
\pi a_{k}=(S A) \int_{0}^{2 \pi} f(x) \cos k t d t \quad \text { and } \pi b_{k}=(S A) \int_{0}^{2 \pi} f(x) \sin k x d t .
$$

It is clear that the problem of recovering the coefficients is a generalization of the uniqueness problem and makes sense only for those systems for which the uniqueness theorem is proved. The uniqueness problem for multiple trigonometric series was solved in 1991 by Tetunashvili [32] (see also [21]) and independently in 1993 by Ash, Freiling and Rinne [1]. So the problem of recovering the coefficients has a meaning for the multiple trigonometric system.

A multidimensional generalization of $S A$-integral or any other multidimensional integral which solves the coefficients problem in higher dimensions has not been obtained yet. Nevertheless, the problem can be solved in a roundabout way on the basis of the Tetunashvili method used in [32]. We show here in what way the Tetunashvili method can be used to solve the recovering problem in higher dimensions (see [30]).

Let $\left\{t_{j}\right\}_{j=0}^{\infty}$ represent the one-dimensional trigonometric system so that $t_{0}=1 / 2$, $t_{2 j-1}=\cos j x, t_{2 j}=\sin j x, j=1,2, \ldots$. The rectangular convergence of the $m$ dimensional trigonometric series

$$
\sum_{\mathbf{n}} a_{\mathbf{n}} \prod_{i=1}^{m} t_{n_{i}}\left(x_{i}\right)=\sum_{n_{1}=0}^{\infty} \ldots \sum_{n_{m}=0}^{\infty} a_{n_{1}, \ldots, n_{m}} \prod_{i=1}^{m} t_{n_{i}}\left(x_{i}\right)
$$

is defined in the same way as for the Haar series in terms of convergence of its rectangular partial sums

$$
S_{\mathbf{k}}(\mathbf{x})=\sum_{n_{1}=0}^{k_{1}} \ldots \sum_{n_{m}=0}^{k_{m}} a_{\mathbf{n}} \prod_{i=1}^{m} t_{n_{i}}\left(x_{i}\right)
$$

when $\|\mid \mathbf{k}\| \| \rightarrow \infty$. We say that the series (6) converges iteratively to $f(\mathbf{x})$ if it converges when $m$ infinite sums in (6) are iterated from right to left, i.e., when the series (6) is understood as

$$
\sum_{n_{1}=0}^{\infty}\left(\sum_{n_{2}=0}^{\infty}\left(\ldots\left(\sum_{n_{m}=0}^{\infty} a_{n_{1}, \ldots, n_{m}} \prod_{i=1}^{m} t_{n_{i}}\left(x_{i}\right)\right) \ldots\right)\right)
$$


We note first that the problem of recovering the coefficients for iteratively convergent trigonometric series can be solved by iterated $S A$-integral in the following way.

THEOREM 3. If the series (6) converges iteratively everywhere on $\mathbf{R}^{m}$ to a finite function $f$, then it is the Fourier series of $f$ in the sense of iterated $S A$-integral, i.e., for each $\left(n_{1}, \ldots, n_{m}\right) \in Z_{0}^{m}$

$$
\pi^{m} a_{n_{1}, \ldots, n_{m}}=(S A) \int_{0}^{2 \pi}\left(\ldots\left((S A) \int_{0}^{2 \pi} f\left(x_{1}, \ldots, x_{m}\right) \prod_{i=1}^{m} t_{n_{i}}\left(x_{i}\right) d x_{1}\right) \ldots\right) d x_{m} .
$$

The following theorem is a special case of the Tetunashvili principal result in [32].

THEOREM 4. A multiple trigonometric series that converges rectangularly everywhere converges iteratively to the same function.

As a corollary from Theorems 2, 3, and 4 we get

THEOREM 5. If the series (6) is convergent rectangularly everywhere on $\mathbf{R}^{m}$ to a finite function $f$ then it is the Fourier series of $f$ in the sense of iterated $S A$-integral, i.e., its coefficients $a_{\mathbf{n}}$ can be computed by (7).

Although the question of defining a multiple integral to solve the same problem is left open, we get from theorem 4 that if it is known that the sum of rectangularly convergent series (6) is integrable in the sense of a multiple integral for which the Fubini theorem holds and for which the corresponding one-dimensional theorem holds, then the series (6) is the Fourier series of its sum in the sense of this integral. In particular the Fubini theorem holds for multiple Henstock integral defined with respect to the full interval basis $\mathcal{B}$ (see [19]). So we get

THEOREM 6. Let $\mathcal{B}$ be the full interval basis. If series (6) converges rectangularly everywhere on $[0,2 \pi]^{m}$ to an $H_{\mathcal{B}}$-integrable function $f$ then it is the Fourier-Henstock series of $f$.

\section{References}

[1] J. M. Ash, C. Freiling and D. Rinne, Uniqueness of rectangularly convergent trigonometric series, Ann. Math. 137 (1993), 145-166.

[2] B. Bongiorno, L. Di Piazza and D. Preiss, Infinite variation and derivatives in $\mathbf{R}^{n}$, J. Math. Anal. Appl. 224 (1998), 22-33.

[3] B. Bongiorno, L. Di Piazza and V. Skvortsov, A new full descriptive characterization of the Denjoy-Perron integral, Real Anal. Exchange 21 (1995/96), 656-663.

[4] B. Bongiorno, L. Di Piazza and V. Skvortsov, On continuous major and minor functions for the n-dimensional Perron integral, Real Anal. Exchange 22 (1996/97), 318-327.

[5] P. Bullen and R. Vyborny, Some applications of a theorem of Marcinkiewicz, Canad. Math. Bull. 43 (1991), 165-174.

[6] J. C. Burkill, Integrals and trigonometric series, Proc. London Math. Soc. (3) 1 (1951), 46-57.

[7] A. Denjoy, Leçons sur le calcul des coefficients d'une série trigonométrique, Paris, 1941-49. 
[8] H. Feizic, C. Freiling and D. Rinne, Two dimensional partitions, Real Anal. Exchange 19 (1993-94), 540-546.

[9] M. de Guzmán, Differentiation of Integrals in $\mathbf{R}^{n}$, Lecture Notes in Math. 481, SpringerVerlag, 1975.

[10] B. Golubov, A. Efimov and V. Skvortsov, Walsh Series and Transforms, Kluwer, 1987.

[11] R. Henstock, The equivalence of generalized forms of Ward, variational, Denjoy-Stieltjes, and Perron-Stieltjes integrals, Proc. London Math. Soc. 10 (1960), 281-303.

[12] R. Henstock, Definitions of Riemann type of variational integrals, Proc. London Math. Soc. 11 (1961), 402-418.

[13] R. Henstock, The General Theory of Integration, Clarendon Press, Oxford, 1991.

[14] R. D. James, A generalized integral (II), Canadian J. Math. 2 (1950), 297-306.

[15] J. Kurzweil, Generalized ordinary differential equations and continous dependence on a parameter, Czech. Math. J. 7 (1957), 418-446.

[16] J. P. Kahane, Une théorie de Denjoy des martingales dyadiques, Enseign. Math. (2) 34 (1988), 255-268.

[17] J. Marcinkiewicz and A. Zygmund, On the differentiability of functions and the summability of trigonometric series, Fund. Math. 26 (1936), 1-43.

[18] K. M. Ostaszewski, Henstock integration in the plane, Mem. Amer. Math. Soc. 353 (1986).

[19] W. F. Pfeffer, The Riemann Approach to Integration, Cambridge Univ. Press, 1993.

[20] D. Preiss and B. S. Thomson, The approximate symmetrc integral, Canadian J. Math. 41 (1989), 508-555.

[21] D. Rinne, Rectangular and iterated convergence of multiple trigonometric series, Real Anal. Exchange 19 (1993-94), 644-650.

[22] S. Saks, Theory of the Integral, Dover, New York, 1964.

[23] V. A. Skvortsov, Haar series with convergent subsequences of partial sums, Dokl. Akad. Nauk SSSR 183 (1968), 784-786; English transl.: Sov. Math. Dokl. 9 (1968), 1464-1471.

[24] V. A. Skvortsov, The Marcinkiewicz-Zygmund integral and its relations to the Burkill SCP-integral, Vestnik Moskov. Univ. Ser. I Mat. Mekh. 27 (1976), No. 5, 78-82.

[25] V. A. Skvortsov, Generalized integrals in the theory of trigonometric, Haar and Walsh series, Real Anal. Exchange 12 (1986/87), 59-62.

[26] V. A. Skvortsov, A Perron-type integral in abstract space, Real Anal. Exchange 13 (1987/88), 76-79.

[27] V. A. Skvortsov, On the Marcinkiewicz theorem for the dyadic Perron integral, Mat. Zametki 59 (1996), 267-277.

[28] V. A. Skvortsov, A variational measure and sufficient conditions for the differentiability of an additive interval function, Vestnik Moskov. Univ. Ser. I Mat. Mekh. 1997, no. 2, $55-57$.

[29] V. A. Skvortsov, Variation and variational measures in integration theory and some applications, J. Math. Sci. (New York) 91 (1998), 3293-3322.

[30] V. A. Skvortsov, On the problem of recovering the coefficients of convergent multiple trigonometric series, Atti Sem. Mat. Fis. Univ. Modena 48 (2000).

[31] V. A. Skvortsov and B. S. Thomson, Symmetric integrals do not have the Marcinkiewicz property, Real Anal. Exchange 21 (1995/96), 510-520.

[32] Sh. T. Tetunashvili, On some multiple function series and the solution of the uniqueness problem for Pringsheim convergence of multiple trigonometric series, Mat. Sbornik 182 (1991), 1158-1176. 
[33] B. S. Thomson, Derivational bases on the real line, I, II, Real Anal. Exchange 8 (1982/83), 67-207, 278-442.

[34] B. S. Thomson, Derivates of intervals functions, Mem. Amer. Math. Soc. 452 (1991).

[35] B. S. Thomson, Symmetric Properties of Real Functions, Monographs Textbooks in Pure and Appl. Math. 183, Marcel Dekker, 1994.

[36] B. S. Thomson, Some properties of variational measures, Real Anal. Exchange 24 (1998/99), 845-854.

[37] A. Zygmund, Trigonometric Series, Cambridge Univ. Press, 1968. 